\title{
Non-flagellate Pseudomonas aeruginosa in pathological material
}

\author{
F. W. LEE \\ From the Department of Pathology, Westwood Hospital, Beverley, Yorkshire
}

SYNOPSIS Three isolates of non-flagellate Pseudomonas aeruginosa obtained from pathological material of human cases are described. Two were found in urinary tract infections and the third in a respiratory infection. They were shown by typing methods to be distinct strains.

Three isolates of Pseudomonas aeruginosa are described. Two were obtained from urinary tract infections and one from a respiratory infection. All isolates were found to be non-motile and were subsequently shown to be non-flagellate by electron microscopy. The purpose of this paper is to report the characteristics of these uncommon strains which were all isolated as a result of routine microbiological investigation of pathological specimens.

\section{Case Reports}

CASE 1

In a 70 -year-old man with a urinary tract infection of some 13 years' standing, due to Ps. aeruginosa, despite several courses of antibiotics, the condition has persisted. A fresh midstream specimen of urine was examined and contained more than 1000 leucocytes $/ \mathrm{mm}^{3}$, 120 red blood cells $/ \mathrm{mm}^{3}$, a trace of protein, no glucose, and the $\mathrm{pH}$ was acid. The culture yielded a confluent growth of a mucoid colony resembling Klebsiella species but later identified as Ps. aeruginosa.

\section{CASE 2}

A 60-year-old female, who has been a long-term psychiatric case, developed an infection of the urinary tract during a recent term in hospital. A fresh midstream urine was found to contain more than 1000 leucocytes $/ \mathrm{mm}^{3}$, no red blood cells, protein, no glucose, and the $\mathrm{pH}$ was acid. A confluent growth of Ps. aeruginosa was obtained.

\section{CASE 3}

A 73-year-old man was admitted to hospital with bronchopneumonia. Sputum culture showed a confluent growth of Ps. aeruginosa. Despite antibiotic and other treatment, the patient died three Received for publication 8 August 1973. days after admission. The cause of death was bilateral bronchopneumonia due to chronic bronchitis and emphysema.

\section{Materials and Methods}

Midstream specimens of urine from cases 1 and 2 and sputum from case 3 were examined within one hour of collection. Primary isolation of the pathogens was obtained by aerobic incubation overnight at $37^{\circ} \mathrm{C}$ of culture plates inoculated with one loopful of the specimen. MacConkey agar was employed foro the urine specimens and nutrient blood agar for the sputum.

The organisms were designated 1,2 , and 3 corresponding to cases 1,2 , and 3 . Repeat specimens were requested and the organisms as described were recovered.

\section{MICROSCOPICAL INVESTIGATIONS}

Gram-stained films from 24-hr nutrient agar cultures were prepared. Electron microscopy was performed on 24-hr peptone water cultures allowed to dry on a carbon-coated copper grid and negatively stained with $2 \%$ uranyl acetate. A hanging-drop preparation of a 24-hr peptone water culture was examined for motile organisms.

\section{CULTURAL AND PHYSIOLOGICAL}

CHAR ACTERS

Colonial morphology and pigment formation on nutrient agar was recorded after 24-hr incubation. Anaerobic growth was tested for by incubation of blood agar cultures in a McIntosh and Fildes jar. The property of the strains to grow at $42^{\circ} \mathrm{C}$ was investigated by serial inoculation into nutrient broth, prewarmed to $42^{\circ} \mathrm{C}$. Growth on $0.03 \%$ cetrimide nutrient agar was determined by inoculation with one loopful of culture in peptone water and incubation for $24 \mathrm{hr}$ at $37^{\circ} \mathrm{C}$. 
FURTHER BIOCHEMICAL AND OTHER TESTS

The following tests were performed as described by Cowan and Steel (1970). The particular method used when alternatives are given is indicated in parenthesis: oxidase; catalase (method 1); oxidation of gluconate (method 2); arginine decarboxylase, nitrate reduction, fermentation of glucose ammonium salt sugars, gelatin liquefaction (method 2).

\section{PYOCINE TYPING}

The method of Govan and Gillies (1969) was employed.

\section{PHAGE AND SEROLOGICAL TYPING} These were kindly performed at the Cross Infection Reference Laboratory, Central Public Health Laboratory, by Dr T. M. Parker.

\section{Results}

The three isolates were found to be Gram-negative rods which were non-motile by the hanging drop technique. Electron microscopy was used to study bacterial morphology because of its availability and advantages over staining methods. A known motile strain was also examined, exhibiting the typical single flagellum. The three strains being studied were found to be non-flagellate. Other results are shown in tables I and II.

\begin{tabular}{llll}
\hline $\begin{array}{l}\text { Cultural and Biochemical } \\
\text { Characteristics }\end{array}$ & Strain & & \\
\cline { 2 - 4 } & \multicolumn{1}{l}{} & 2 & 3 \\
\hline Anaerobic growth & - & - & - \\
Green pigment & + & + & + \\
Trimethylamine odour & + & + & + \\
Growth at 42.C & + & + & + \\
Growth on cetrimide agar & + & + & + \\
Oxidase & + & + & + \\
Catalase & + & + & + \\
Nitrate reduction & + & + & + \\
Glucose & + & + & + \\
Arginine & + & + & + \\
Gluconate & + & - & - \\
Gelatin liquefaction & + & + & Mucoid \\
Colony & Mucoid & Rough & - \\
\hline
\end{tabular}

Table I Cultural and biochemical properties of the non-flagellate Ps. aeruginosa strains

\begin{tabular}{llll}
\hline Typing Methods & Strain & & \\
\cline { 2 - 4 } & 1 & 2 & 3 \\
\hline Pyocine & 5 & lc & lc \\
Phage & No lysis & Lysis + & No lysis \\
Serological & 4 & 9 & not typable \\
\hline
\end{tabular}

Table II Results of typing methods

\section{Discussion}

Ps. aeruginosa is described by Cruikshank (1965) as Gram-negative rods actively motile by virtue of polar flagellae rarely numbering more than three.

I understand that non-flagellate strains have been induced by artificial means (Pitt, 1973) but their isolation from clinical material is rare. Jessens (1965) found less than $6 \%$ of 354 isolates of the organism from various sources, including pathological specimens, to be non-motile and shown to be nonflagellate by Leifson's staining technique.

The mucoid colonial form is rarely isolated from the urinary tract, being more commonly implicated in respiratory infections. Martin (1973) reported four such strains from a total of 87 isolates from urinary infection. It was the chance finding of a mucoid colony from case 1 , subsequently identified as non-flagellate $P s$. aeruginosa, that led to the examination of other isolates of this organism from all types of infection. To date, two further strains have been found, one from a urine specimen and the other from a sputum.

Some 20 other isolates have proved to be motile. As this study is recent, I cannot suggest how long these strains have been present in these infections; nor is it possible from the small number of organisms studied so far to assess the incidence of non-flagellate Ps. aeruginosa in pathological materials.

It is interesting to note that the three strains described here have been shown by typing methods to be distinct organisms.

Reference to the occurrence of non-flagellate $P s$. aeruginosa appears to be rare. It is intended to further the investigations initiated by this work in order that the incidence of non-flagellate strains in human infections may be assessed. In this connexion I would be interested to learn from other workers of similar isolates.

I should like to thank Drs K. H. Sugars, J. Johnston, and T. W. G. Kinnear for clinical information, Mrs J. E. Mundy for electron microscopy and photography, and Mr T. L. Pitt.

\section{References}

Cowan, S. T., and Steel, K. J. (1970). Manual for the Identification of Medical Bacteria, pp. 146-165. University Press, Cambridge.

Cruickshank, R. (1965). Medical Microbiology, 11 th ed., p. 260 Livingstone, Edinburgh.

Govan, J. R. W., and Gillies, R. R. (1969). Further studies in the pyocine typing of Pseudomonas pyocyanea. J. med. Microbiol., 2, 17-25.

Jessen, O. (1965). Pseudomonas aeruginosa and other Green Fluorescent Pseudomonads. Munksgaard, Copenhagen.

Martin, D. R. (1973). Mucoid variation in Pseudomonas aeruginose induced by the action of phage. J. med. Microbiol., 6, 111-118. Pitt, T. L. (1973). Personal communication. 